\title{
GANGRENA DE LAS EXTREMIDADES EN NIÑOS RECIEN NACIDOS
}

\author{
Drs. HUMBERTO GARCES y ELIANA ROSENBLUT
}

\author{
Cánedra de Pediatria del Prof. A. Scroggie. Hospital "Roberto del Río".
}

Las lesiones gangrenosas de las extremidades son poco frecuentes en el recién nacido. En una revisión bibliográfica muy completa hecha por Bazán" en 1954, concluye que hasta esa fecha se han publicado 54 casos, incluyendo el suyo. Parece ser más común en niños prematuros que en niños de término.

Según Allen, Barker e Hines (citados por Askue ${ }^{1}$ la oclusión arterial que produce la gangrena, puede ser el resultado de un atrombosis, de una embolía, o de un simple espasmo, de donde resultaría la distinta evolución del cuadro clínico.

La etiología de este proceso es variada. Si la gangrena está presente en el momento del nacimiento, hay que pensar en una brida amniótica que haya producido la compresión de la extremidad, y que puede llegar hasta la amputación de ella.

Si la lesión aparece después del nacimiento puede originarse por:

1) Traumatismo obstétrico en partos prolongados, en especial cuando hay procidencia o retención de un miembro, que es comprimido entre la cabeza del niño y la pelvis materna, originando, en la extremidad afectada, el adosamiento de las paredes de los vasos sanguíneos, y la alteración consiguiente de la íntima, seguida o no de la formación de trombos que obstruyen dicho lúmen.

2 . Cardiopatías congénitas, que facilitan la formación de trombos, en especial el ductus persistente. Nuestro caso $\mathrm{N}^{9} 2$ tenía una cardiopatía congenita.

3) Sepsis, en que embolias microbianas entran a la gran circulación y se detienen en las arterias de las extremidades. Esta etiología estuvo presente en dos de nuescasos.

4) Trombosis de las arterias umbilicales, que después del nacimiento pueden dar origen a coágulos que se extienden a las hipogástricas o iliacas externas. (Gross, citado por Moura ". Nuestro caso No 3 se originó en una trombosis de los vasos umbilicales que se produjo como consecuencia de una exanguineo transfusión practicada por una Eritroblastosis fetal.
La mayoría de los casos descritos en la literatura, se han desarrollado en casos de sepsis graves del recién nacido y tienen, por consiguiente, un grave pronóstico.

Producida la obstrucción arterial, se genera una alteración circulatoria en la parte más distal, de la extremidad, que se inicia por un color rojo violáceo, que poco a poco va tomando un tinte más obscuro, hasta llegar a negro. La extremidad, inicialmente engrosada y edematosa, presenta Iuego un surco que delimita la parte sana, con buena circulación, de la lesionada que poco a poco va momificándose $y$ termina por eliminarse. No es raro observar la formación de flictenas epidérmicas, con contenido serohemorrágico en las partes que van a ir a la necrosis.

La gangrena puede producir gran toxemia que lleva a la muerte, aunque se recurra a la amputación quirúrgica precoz, como en el caso de Brown y Smith " en el que, a pesar de haberse practicado una amputación al $2^{y}$ día, se produjo el fallecimiento. Otros son de naturaleza más benigna, como el citado por Stadler ${ }^{7}$ : se trata de un recién nacido que presentó un trastorno circulatorio de ambos pies desde el 1.er día de la vida, cuya etiología no se pudo precisar q que mejoró completamente con un tartamiento conservador. Prokablemente pudo haberse producido por un espasmo transitorio de las arterias.

Heller y Alvari (citados por Foster y Tavlor ${ }^{8}$ sustentan una teoría que explicaría la patogenia y evolución de estas lesiones reversibles. de pronóstico benigno: las paredes de las arterias más pequeñas se adosarían, depositándose fibrina que aglutinaría aún más las íntimas de los vasos: cambios de presión pueden hacer que la circulación sanguinea se restablezca antes que aparezcan cambios irreversibles.

El tratamiento debe ser eminentemente general $\mathrm{y}$ se encaminará a:

1) Colocar al niño en las mejores condiciones, que aumenten su vitalidad, evitando traumatizar el miembro afectado;

2) Uso de antibióticos para prevenir o curar la infección; 
3 ) Aumentar la circulación colateral mediante el uso de vasodilatadores;

4 I Impedir el progreso de la trombosis mediante anticoagulantes; y

5) Ussar la amputación como último recurso, cuando se compromete el estado general y la vida del niño por la toxemia. Los territorios necrosados van espontánexmente a la gangrena seca que termina рог su eliminación, cuando no se recupera por medio de la circulación colateral.

\section{CASOS CLINICOS}

CASO N: I. V. C. S. (Obs. $\mathrm{N}^{0} 143472$ ).

Antectdentes hredirarius: Sin importancia.

intecedonies personctes: Nació de embarazo de 8 mosìs. en parte noimal, espontánco. atendido en Maternidad. Pesó 2.700 gramos. Durante los primeros 15 dias fuć alimentada a pecho y posieriormente con Eiledón.

Enformedad aciuat: 4 dias antes del ingreso, a los 20 dias de cdad, presenta mal estado general con vómitos y deposiciones licuidas frecuentes. cuadto al que se sgrega 2 diats despulis. enrojecimiento cianótico de ambos pies.

lixamen fisico: A su ingreso al Servicio se aprecia nin̆a mul gtave, marsado enfraimiento, pésimo estado nutritivo (2.300 gramos), panículo adiposo casi ausente. Hipotonía muscular. Escleredema gencra!izado. Sersorio umbocado. estado de coma, no liora. Fontanclas abjetlas. deprimidas. Notable palidez de la cara. Macosas secas. Respiración superficial, Estertores subcrepitantes de tipo distejectísico en ambas regiones paravertebrales y bases. Tonos cardiacos muy apagados. Cicatriz umbilical sana. Paredes abdominales infiltradas y rigidas, por sl escleredema. Hígado a uno Y medio traves del reborde costal, de cousistencia fitme. Se palpa el bayo. Extremidades con marcado esclercdema. enftiamionlo $y$ cianosis. En ambos pies $y$ (n) ia mano deretba presenta la piel de aspecto equimólito. con ampollas con tontenido hemorrágico.

Se le practica un hemograma que tevela:

Hematics: 6.200 .000 por mm.: Hemoglobins: 1]5 Tr: Leucacitos: 65.900 por mm.; Linfocitos: 24: Segmentados: 36: Bacili lormes: 29: Juveniles: 3; Mielocíos: 2.

Evolución: Se trata con Tetramicina. Penicilina $y$ Estreptemicina durante 10 dias. Se alimenta con lecte humana mediante sonda. Se punciona las ampoltas descritas pata examen bactcriológico, cuyo culcivo revela microcopus piogenus alhus.

Durante los pritheros dias del ingreso se mantiene in estado grave. presencando temperatuta del lipo sciptico. La epidermis de anbos pies y muno izçuierda macetada. se ha e'iminado casi completamente. incinyendo las añas y dejando et dermis al descubierto: a] mismo ticmpo en el límite con la piel sana. en ambas piernas. comienza a aparecer un surco que paulalinamente se profundiza. Las lesiones de pies y manos loman aspetto gangrenoso. de mal olor y los dedos se momifican. A módidil que las lesiones se valn delimitando por el surso. descrito va mejorando el estado general. (Figura $N^{n} 1$ ).

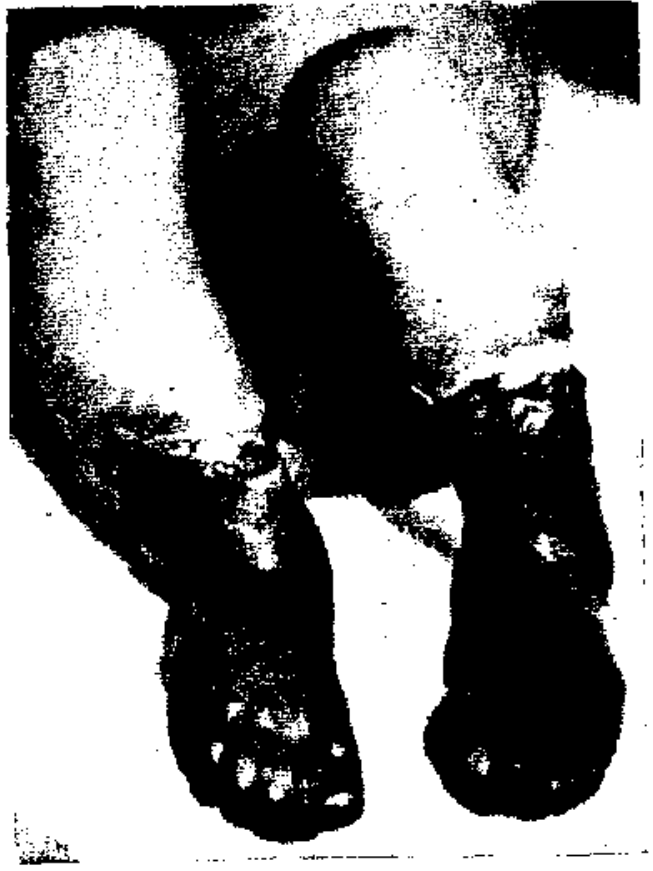

Figura $V^{\prime}$ 1: Gangrena simétrica de ambos pies en el caso N!' $T$, donde se puede apreciar un surco biten neto que delimita la parte sana de la pierna.

A $\operatorname{los} 12$ dias de ingreso se elimina cl pie derecho junto con el tercio inferior de la pierna. A los 15 dias se climina el pie izquictdo a la misma altura del derecho, y también todos los dedos de la mano izquierds. Al mes de ingreso los muñones se aprecian limpios, en franca via de cicatrización. El hemograma de control demuestra una reducción de los leucocitos a 8.800. monositos 19. lincocitos 45. segmentados 17 y baciliformes 17 . Tiene anemia de 3.700 .000 con $68 \%$ de hemoglobina, que se trata con transfusiones de sangre.

A1 mes di ingreso ha subido $400 \mathrm{gr}$. de peso $\mathrm{y}$ ha mejorado en forma apreciable ef estado general. Poco d espućs prescnta una diarrea aguda $y$ una bron. coneumonia difusa bilateral, de evolución arrastrada y prolongada. que ceden con antibióticos y plasmoterapia,

Permanece 94 dias en el Setvicio, siendo dada de alta con 3.300 gr. de peso. Los mufiones de ambos miembros inferiores están completamente cicatrizados. de tipo cónico, que es preciso regulatizar posteriormente. Fil muñón de la mano izqaierda tambićn se aptecia cicarrizado. La niña se ha seguido controlando en el Consultorio Externo del Servicio hasta los 2 uños. Su distrofia se ha corregido completamente. En el presente año se tomaron en el Servicio de Ortopedia moldes de yeso de sus muñones, pafa hacerle los aparalos orcopídicos correspondientes.

CASO N:2 M. G. A. (Obs. NN 161351 )

Antecedentes personoles: nació de parto normal espontinco, en stl casa. atendido por una vecina. No se pesó al ajaerer. a los 8 dias pesaba 2 Kg. Alimentada hasta los 15 dias a pecto cada 3 horas. En esa época se agrega to gr. de Eledón 3 veces al dia. 
Enfermedad actual: Ingresa al Serpicio enviada por et Consultorio Externo de Cirugía, en vista de su mal estado general, donde habia consultado por ana hernia inguinal.

Examen físico: Se aprecia una niña pequeña, en pésimas condiciones nutritivas y generales. Peso 2.250 gr.. muy deshidratada, pálida, decaida, febril. Al examen cardiaco se ausculta soplo sistólico suave en el $2^{\circ}$ y $3^{\circ}$ espacio intercostal izquierdo, sin irradiación. Taquicardia, tonos apagados. Higado grande $y$ duro. Bazo no se palpa. Hernia inguialal derecha.

El hemograma, hecho al ingreso, demuestra:

Hematies: 4.000.000 por mm.: Hemoglobina: $78 \%$; Leucocitos: 15.000 por $\mathrm{mm}$.: Monocitos: 8; Linfocitos: 27; Plasmáticos: 1; Baciliformes: 12: Eosinófiles: 1.

Se bace el diagnóstico de Prematnrez. Cardiopatía Congénita. Hernia inguinal derecha. Sepsis.

\section{Evolución :}

Como tratamieato se indica penicilina $y$ estrepromicina, en dosis babituales. El tratamiento con antibióticos se mantiene pot 18 días. Además se agregan transfusiones de sangre y plasma.

a los 2 dias de ingreso se agrava, los tonos catdíacos están más apagados $y$ presenta edema. cianosis y enfriam,ento de la mano derecha, en la cual se desprende la epidermis. Esta lesión evolucionó a la gangrena seca, momificándose y eliminándose las puntas de los dedos, a los 13 dias de ingreso. Casi al mismo tiempo apsitace. una placa equimótica del borde de $l_{1}$ lengua $y$ inftamiento $p$ cianosis de la mano izquierda, que regresan agregando al tratamiento fleboclisis d: suero glucosado c. n $0.5 \%$ de novocaina y terramicina endovenosa, quedando solo en la mano pequeños focos de necrosis de la piel, que se eliminan, liegando posteriormente a la restitución compkta. (Figura N? 2).

El estado general deficiente, mejora a partir de los 20 dias del ingreso. Queda afebril a los 13 días, co.acidiendo con la eliminación de los dedos gangrenados. Está aperente, desaparece el edema y al cxamen cardíaco persiste el soplo sistólico antes descrito. Poscstiotmente se opera de sn hernia ingainal y se da ds alca después de 91 días con $4.200 \mathrm{gr}$. de peso.

\section{CASO $N^{0} 3 \mathrm{M}$. G. M. (Boletin $\mathrm{N}^{\circ} 4626$ ).}

Antecedentes heteditarios: Padre de 42 años, aparentemente sano, sin antecedentes mórbidos. Madre de 32 años, aparentemente sana, ha tenido 9 embarazos con niño vivo, de los cuales viven 6: 2 fallecidos en la segunda infancia $y$ el tercero, anterior al caso que nos preocupa, nació con jetericia intensa y falleció 15 dias después del nacimiento.

Antecedentes personales: Nacida de embarazo de término, de evolnción normal, en la Maternidad del Hospital "San Borja". Pesó 3.000 gr. al nacer.

Enfermedad actual: A las 6 horas del nacimiento presenta tinte ictérico intenso de la piel y mucosas. $A l$ examen se aprecia hepatomegalia y esplenomegalia. Por el antecedente y el cuadro clinico, se hace el diagnóstico de Eritroblastosis, y se te pracican los siguientes exíneeves:

10 Determinación de grupos sanguineas:

Madre: B. -R.h (

Niño: A.R.h $(+)$.

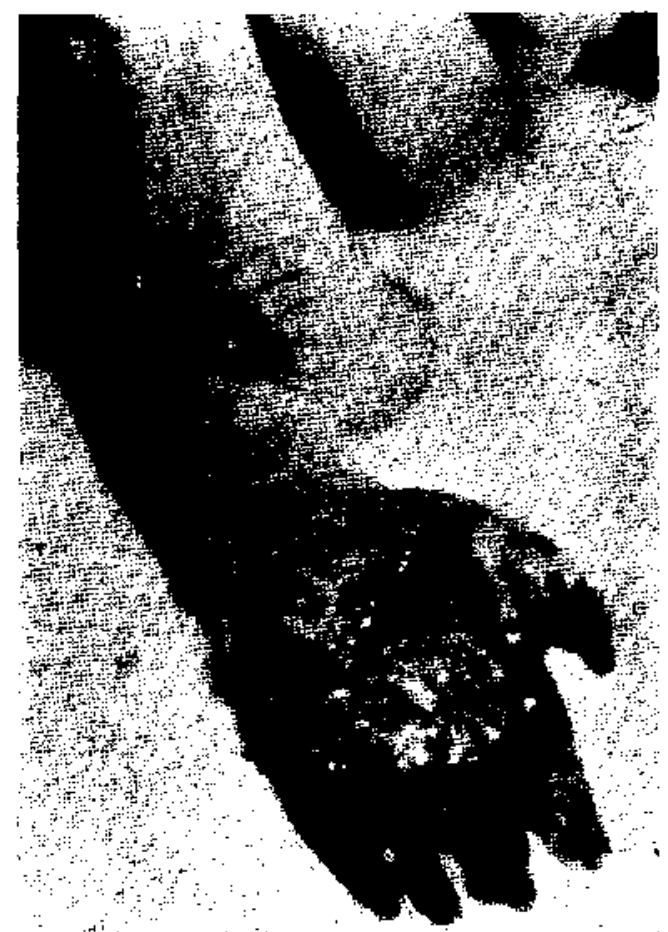

Figura No 2: Gangrena de la mano derecha en el caso No 2. Nótese el aspecto edematoso y necrótico de la piel y el desprendimiento de la epidermis en la palna de la mano.

$2^{0}$ Test de Coombs directo: positivo.

39 Bilitrubinemia en sangre del cordón: Ditecta $3,5 \mathrm{mgr} \%$. Indirecta $33,5 \mathrm{mgr} \%$. Total $37 \mathrm{mgr} \%$. $4^{\circ}$ Hemograma: Hematoctito, $50 \%$; Hemoglobina. $14 \mathrm{gr} . \%$; Reticalocitosis, $20 \%$; Eritroblastemia, $134 \%$.

Micrositosis y. microesferocitosis: Juyeniles 4; Baciliformes, 46; Segmentados, 32: Linfocitos, 18.

Evolución:

Estos exámenes compraeban el diagnóstico de Enfermedad Hemolitica del recién nacido por incompatibilidad al factor sanguíneo $R$. Se decide practicar una exanguino-transfusión, que se efectúa 7 horas después del nacimiento.

El niño la tolera mal. Durante la sustitución tiene vómitos, cianosis y polipnea, por lo cual se debe sospender a los 390 cc. de sangre. (Se extraen $410 \mathrm{cc}$.). Después del recambio se indica calentamiento, oxigeno. penicilina y estreptomicina. 24 horas después del recambio ( 31 después del nacimiento) el niño está quejumbroso, con aleteo nasal, ba aumentado el tinte ictérico de la piel, que aparece deshidratada. Llama la atención el edema del dorso del pie izquierdo. cuyos dedos se aprecian intensamente cianóticos, frios y tumefactos. En el ortejo mayor se agregan zonas vesicalosas. Esta lesión se interpreta como una embolía consecutiva a una trombosis, cuyo punto de partida podria ser la vena umbilical o un coágulo (es posible que un coágulo del matraz se deslice a través del filtro, si si coma en cuenta que aspira con cierta presión! 
Se hace el diagnóstico de gangrena de los dedos del pie izquierdo. A las 36 horas la bilirrubinemia de control es: Directa: $21,3 \mathrm{mgr}$. \% : Indirecta: $63,6 \mathrm{mgr} . \%$. Total, 84,9 mgr \%. Un control de Hemoglobina da 12 gr. $\%$.

Como tratamiento se indica Penicilina, Estreptomicina y Terranicina que se mantiene por 10 dias. Fleboclisis de suero glucosalino $2 / 3$ y plasma. Inyección de novozaína al $2 \%$ en la arteria tibial con objeto de producir vasodilatación. Se alimenta con Eledón en cantidades progresivas. 3 dias respués del recambio, aún persistiendo el tince ictérico de la piel y mucosas, el bazo se palpa más chico. Disminuye el edema del dorso del pie, al mismo tiempo que las lesiones descritas en los dedos se delimitan mejor, pareciendo nijoras la circulación. En los dias siguientes las lesiones descritas regresan apreciablemente, pero ya se observa un surco que va delimitando las últimas falanges del ortejo mayor $y$ de los ortejos ségundo $y$ tercero que van a una futura eliminación. Se da de alta a los 10 días en buenas condiciones gerietales. (Figura $N^{0} 3$ ).

Voelve al control 10 dias después del alta. No hay ictericia, pero se aprecia una intensa palidez de piel $y$ macosas. Se allmenta bien. Un control de hemoglobina da $55 \%$, por to que se practican 3 transfusiones de sangre. Al examen se comprabia la aliminación de la iltima falange del ortejo mayor, al mismo tiempo que el sarco que delimitaba la última falange del segundo

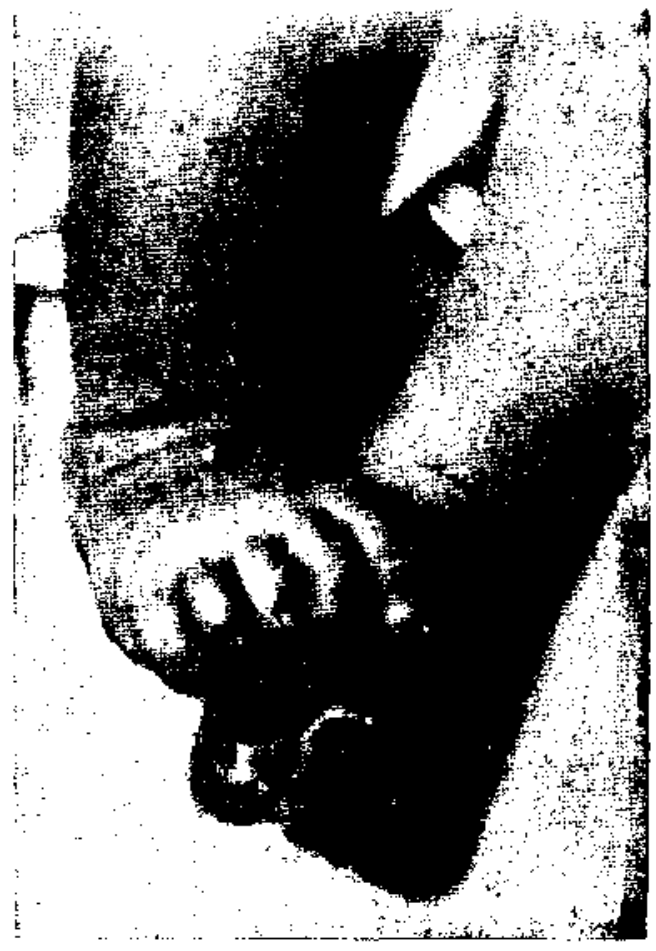

Figura N" 30 Gangrena de los tres primeros ortejos đej pie izquierdo en el caso $\mathrm{N}^{*} 3$. La lesión es más incensa a nivel del ortejo mayor, que tiene un color más obscuro. y tercer ortejo se ha profundizado. A los 30 dias de edad, se han eliminado estos últimos dedos y los muñones están cicatrizados, en buenas condiciones.

\section{RESUMEN}

Se describen 3 observaciones clínicas de niños recién nacidos, que presentaron gangrena de las extermidades.

En el caso $N$ i se trata de una sepsis grave, con gangrena simétriça de los miembros inferiores y una mano. El punto de partida de las embolias no es posible precisarlo, El tratamiento intenso con antibióticos permitió salvar la vida de la niña, que perdió los dos pies hasta el $1 / 3$ inferior de las piernas y varios dedos de la. mano izquierda.

En el caso $N^{9} 2$ se trata también de una sepsis que produce una gangrena de la mano derecha, que Iléga a la eliminación de las puntas de los dedos y de una lesión semejante, pero de menor intensidad, de la mano izquierda, que cede con vasodilatadores; restituryéndose en buenas condiciones.

En el caso No 3 se trata de un trombo de punto de partida umbilical producido por la exanguino-transfusión, en un caso de Eritroblastosis fetal, que obturó el territorio de la arteria pedia, con pérdida de tres ortejos del pie izquierdo.

\section{SumMarY}

\section{Gangrene of the eXtremities IN THE NEWBORN INFANT}

A description is done on 3 clinical cases of gangrene of the extremities in the nowborn infant.

The case $\mathrm{N}^{\circ} 1$, had a severe sepsis, with symmetrical gangrene of the feet and one hand. The point of departure of the embolism couldnot be fixed. Intensive treatment with antibiotics allowed us to save infant's life, who lost both feet up to the legs and several fingers in the left hand.

The case $\mathrm{N}^{\circ} 2$, had also a sepsis, which produced a gangrene af the rigth hand, ending in the loss of the utmost of the fingers. A similar damage, but with less intensity, was observed in the left hand, in which a complete recession was obtajned with the use of vasodilators. 
The case $N^{3} 3$ had a thrombus of point of diparture umbilical, following echangetransfusion in a case of Erytroblastosis, who blocked the artery dorsalis pedis, with lost of 3 toes of the left foot.

\section{Bibliografa}

I-ASKUE, W. E. Y WONG, R. - "Gangrene of the extremitles at the newborn infant". J. Pedlat 40: 588: 1952 .

2-BAAN, C. - "Ganurena del miembro superior en el rectên nacldo". El Recién Nactdo, 2, 53; 1054.
3.-BROWN R. J. y SMITH, S. R. - "Gangrene of the foreann in the netwborn". Arsh. Dis. Chlld. 26 ; 574; 1952

4. CARITAT, J., SOLOVEY, G, y BONABA, J. M. "Un caso de baritrena aséptica del miembro inferlor producida in litero". Arch. Pedlat, del Uruguay; 16 ;614; 1945 .

5.-HANSEN, J. D. - "Gangrène ana thrombosts in en infant with comgential heart disease". Arch. DIs. Childh.: $24: 148 ; 1949$,

6.-MOURA I. - "Gangrens de extremldades en recten nacido". Jourual de Ped. 17:161: 1952.

7.-STADLER, H. E. - "NeonataI Symmetrical pedal kangrene with complete recession". J. Pediat $43: 447$; 1953.

8.-FOSTER W. P y TAYLOR, R C - "Gangrene 1n congenital Syphtils", Arch. Dis. Obildn. $24: 152: 1849$. 